\title{
Effects of Web-Based Supervisor Training on Job Stressors and Psychological Distress among Workers: A Workplace-Based Randomized Controlled Trial
}

\author{
Norito Kawakami, Soshi Takao, Yuka Kobayashi and Akizumi Tsutsumi
}

Hygiene and Preventive Medicine, Okayama University Graduate School of Medicine, Dentistry and Pharmaceutical Sciences, Okayama, Japan

\begin{abstract}
Effects of Web-Based Supervisor Training on Job Stressors and Psychological Distress among Workers: A Workplace-Based Randomized Controlled Trial: Norito KawaKami, et al. Okayama University Graduate School of Medicine, Dentistry and Pharmaceutical SciencesUsing workplaces as a unit for randomization, a randomized controlled trial was conducted to determine the effects of web-based supervisor training on the subject of worksite mental health on job stressors, supervisor support and psychological distress among subordinate workers. A total of eight workplaces of a sales and service company were randomly assigned to either training workplaces or non-training workplaces. Supervisors $(n=23)$ at the training workplaces participated in web-based self-learning training on worksite mental health; supervisors $(n=23)$ at the nontraining workplaces did not. A total of 81 subordinate workers under the trained supervisors (the intervention group) and 108 subordinate workers under the nontrained supervisors (the control group) completed the Brief Job Stress Questionnaire (BJSQ) at baseline and at a three-month follow-up. No significant intervention effect was observed for any scale of the BJSQ measuring job stressors, supervisor or coworker support, or psychological distress among subordinate workers $(p>0.05)$. The item score of work autonomy changed very little in the intervention group, while it decreased in the control group during the follow-up period, yielding a significant intervention effect $(p=0.02)$. The item score for a friendly atmosphere in the workplace increased in the intervention group, while the score remained stable in the control group, yielding a significant intervention effect $(p=0.02)$. While the
\end{abstract}

Received Jun 13, 2005; Accepted Oct 31, 2005

Correspondence to: N. Kawakami, Hygiene and Preventive Medicine, Okayama University Graduate School of Medicine, Dentistry and Pharmaceutical Sciences, 2-5-1 Shikata-cho, Okayama 700-8558,Japan (e-mail:norito@md.okayama-u.ac.jp) present study failed to show any clear effect of the webbased training of supervisors on reduction of job stressors, it may be useful for maintaining worker autonomy and improving the friendliness of the worksite atmosphere.

(J Occup Health 2006; 48: 28-34)

Key words: Web-based training, Health education, Job strain, Worksite health promotion, RCT, Japan

Previous research has shown that supervisor support, as one of the dimensions of worksite social support, has a beneficial effect on worker health ${ }^{1-5)}$. Supervisors are a source of emotional, informational, and instrumental social support ${ }^{6)}$, as well as being key individuals in preventing job stressors in the work environment ${ }^{7)}$. Studies have shown that a supervisor training program on the subject of worksite mental health and job stress prevention are effective in increasing supervisor skills of listening to subordinate workers ${ }^{8,9)}$, perceived supervisor support ${ }^{10)}$, and decreasing job stressors and psychological distress among subordinate workers ${ }^{11)}$.

Applying an innovative method of education using web-based training, which has been used in occupational health settings ${ }^{12,13)}$, we developed training for supervisors aimed at increasing supervisor support and mental health among their subordinates ${ }^{14)}$. The merits of web-based training compared with traditional lectures and workshops are as follows ${ }^{14)}$. (1) Participants do not have to attend lectures together, which sometimes results in a significant reduction of time on the job and considerable expenses in traveling to the training site. (2) Web-based training provides greater flexibility for participants in that supervisors can access the training at their own pace and at any place and any time they like. (3) Participants may repeat the lessons as many times as needed. (4) The progress made by participants can be monitored by a central personnel office, which controls the entire learning 
process. We previously conducted a randomized control trial and found a significant effect of the web-based supervisor training on maintaining supervisor support, while no clear effect on job overload or psychological distress was observed among subordinates ${ }^{14)}$. In this previous study we randomly assigned supervisors working in the same building to training and non-training groups. However, if the information given by the webbased training was also conveyed to the non-training group, the effect might be underestimated. Furthermore, the previous study did not test the effect of the web-based training on the reduction of job stressors other than job overload, such as job control or interpersonal conflicts.

In the present study, we conducted a randomized controlled trial to determine the effects of web-based supervisor training on selected job stressors, as well as supervisor and coworker support, and psychological distress, among subordinate workers. We used workplaces as a unit for randomization to keep to a minimum possible contamination of the information between trained and non-trained supervisors.

\section{Subjects and Methods}

Study design and subjects

The study was conducted in an office machines sales and service company, which had a total of 251 employees in eight workplaces located in the Osaka and Kobe areas of Japan. Most employees were sales and service workers $(60 \%)$, with a smaller proportion of managers/supervisors $(30 \%)$ and clerks $(10 \%)$. These workplaces were randomly assigned using a random number table, into four training workplaces and four non-training workplaces. In November 2002, all supervisors (section chiefs and work group chiefs) $(n=23)$ of the training workplaces were asked to participate in the web-based supervisor training for one to four weeks. The training was provided from an internet server at the Okayama University Graduate School of Medicine and Dentistry, Japan. Twenty-two of the 23 supervisors completed the four-week training session. At baseline (before the training) and just after completing the web-based training, $19(83 \%)$ of the 23 supervisors in the training group were asked to complete a questionnaire on supervisors' knowledge and attitudes concerning mental health in the workplace. During the same period, the non-training supervisors $(n=23)$ participated in a two-hour training session on a method of relaxation which was provided by a clinical psychologist, instead of the web-based training.

In November 2002, before the beginning of the webbased training for supervisors, all employees were asked to participate in a baseline survey of job stress and mental health. The survey was conducted using internet technology. Employees were asked to access the server at Okayama University Graduate School of Medicine and
Dentistry, complete a web-based questionnaire, and then submit it. Three months after the end of the web-based supervisor training, in February 2003, a similar followup survey was conducted.

The analysis was conducted on an intention-to-treat basis. However, one supervisor in the training group died in the middle of the follow-up, i.e., January 2003. We excluded the seven subordinate workers working under this supervisor from the analysis, considering that there might be a psychological effect of his death on subordinate workers. We further excluded from the analysis those who did not complete baseline or followup surveys. A total of 85 subordinate workers were working in the training group workplaces excluding the one above-mentioned workgroup (intervention group workers), while 114 subordinate workers were working in the non-training group workplaces (control group workers). Among these, 81 (95\%) and 108 (95\%) in the intervention group and control group, respectively, participated in both surveys at the baseline and threemonth follow-up. The numbers of women among these respondents were $28(35 \%)$ and $26(24 \%)$ in the intervention and control groups, respectively. The subjects' average ages were 31.0 (6.5) and $32.0(6.0)$ in the intervention and control groups, respectively, and eight $(10 \%)$ in the intervention group and $10(9 \%)$ in the control group worked 60 or more hours of overtime per month.

The study design and procedure were reviewed and approved by the Human Ethics Committee for Epidemiological Research at the Okayama University Graduate School of Medicine and Dentistry, Japan. We did not obtain written informed consent from supervisors or subordinate workers; however, all employees were told about the study procedure and were clearly informed that participation in the study was entirely voluntary. For ethical reasons, after the study was completed, the webbased training was provided to the supervisors in the control group; and a training session on a relaxation method was provided for those in the training group.

\section{Web-based supervisor training}

We developed a web-based training program for supervisors on the subject of worksite mental health, which is called "E-learning Worksite Mental Health for Supervisors" (Fujitsu Infosoft Technology Co. Ltd., Japan, 2002) ${ }^{14)}$. All supervisors accessed the internetbased training from workplace PCs. Most of the supervisors received the training via workplace PCs while some received it via PCs at home. During the four-week training period, a study coordinator oversaw their progress and encouraged them by e-mail to complete the training. The contents of the web-based training included a variety of topics that supervisors were required to know based on "Guidelines for Promoting Mental Health Care in 
Enterprises" by the Japanese Ministry of Health, Labour and Welfare (formerly the Japanese Ministry of Labour) ${ }^{15}$. It consisted of nine chapters following a general introduction (see Appendix). In the first chapter, trainees read the tragic story of a worker with depression, in which a typical problem related to mental health in the workplace is described. Then, in each chapter, trainees studied a specific topic, and, at the end of each chapter, they answered four to five quizzes to confirm their understanding. The average time to complete the entire training was three to five hours.

Assessment of supervisor's knowledge and attitudes to worksite mental health

The supervisors' knowledge and attitudes concerning mental health in the workplace among supervisors in the training group were assessed using an e-mail questionnaire at the baseline and just after completing the web-based training. It consisted of eight questions on the following issues: (a) understanding how to listen to subordinates; (b) positive attitudes toward listening to subordinates; (c) understanding how to provide support to a subordinate who was returning to work after experiencing mental health problems; (d) understanding how to work with occupational health professionals; (e) understanding possible sources of stress in the work environment; (f) positive attitudes to improving the work environment to prevent psychosocial stress; $(\mathrm{g})$ understanding the nature of stress and ways to deal with it; and (h) awareness of and coping with their own stress. The questions were asked so that the participants could use a four-point response system from 1, which indicated "not at all" to 4, which indicated "very well or very much".

Assessment of job stressors, worksite support and psychological distress

The primary outcome in the present study was job stressors and the secondary outcomes were worksite social support and psychological distress. We used the Brief Job Stress Questionnaire (BJSQ) ${ }^{16)}$, a 57-item multidimensional job stress questionnaire using a 4-point Likert-type response option (from "strongly agree"=4 to "strongly disagree"=1), to measures job stressors, worksite support, and psychological distress of workers. For job stressors, we calculated scores of quantitative job overload (three items), qualitative job overload (three items), physical demands (one item), job control (three items), skill underutilization (one item), interpersonal conflict (three items), poor physical environment (one item), suitable job (one item), rewarding job (one item), as well as scores for the individual items constituting these scales. Supervisor support and coworker support were measured by using three-item scale scores, ranging from 3 to 12, for which a higher value was indicative of greater supervisor or coworker support. An 18-item scale from the BJSQ was used to measure the psychological distress of subordinate workers, yielding a score ranging between 18 and 72, for which a high value was indicative of high psychological distress. We modified the original scoring scheme so that a high score indicated a high tendency of an item or scale content (e.g., a higher score for quantitative job overload was indicative of the presence of high job overload). These scales showed acceptable to high levels of internal consistency reliability (Cronbach's alpha at baseline survey, 0.54 for interpersonal conflict, $0.65-0.75$ for other multiple-item job stressor scales, $0.81-0.84$ for worksite support scales, and 0.92 for psychological distress).

\section{Statistical analysis}

We could not set a specific threshold value to endorse a meaningful intervention effect, because quantitative information was still lacking for determination of such a value for job stressors and other outcome measures that have a clinical or practical impact. Average item scores for knowledge and attitude toward worksite mental health were compared between the baseline and immediately after completing the web-based training among 16 supervisors in the training group (paired $t$-test). The average scores of job stressors, supervisor support, coworker support, and psychological distress among subordinate workers were compared for the two groups (the intervention and control groups) and time (at baseline and at three-month follow-up). The intervention effect was tested by examining the interactive effect between the intervention and control groups and between the baseline and three-month follow-up by using repeated analysis of variance (ANOVA). The statistical analysis was conducted using the SPSS version 10 statistical package (SPSS Inc., Chicago, U.S.A.).

\section{Results}

\section{Change in supervisor's knowledge and attitude}

A comparison of knowledge and attitude toward worksite mental health at baseline and just after completing the web-based training indicated that understanding how to listen to subordinates, how to support workers who are returning to work after experiencing mental health problems, how to work with occupational health professionals, and how to deal with stress, significantly increased after the web-based training (Table 1).

\section{Effects on job stressors}

No significant intervention effect was observed for any job stressor scale score (Table 2, $p>0.05$ ). However, when we looked at individual items, the score of the item on worker autonomy for deciding the order of, and ways to do jobs, remained stable during the follow-up period among the intervention group, but decreased among the 
Table 1. Comparison of average scores (standard deviations, SDs) for knowledge and attitude on worksite mental health at baseline and immediately after the training among supervisors $(n=19)$ who took the web-based training course

\begin{tabular}{lcrrrr}
\hline \multirow{2}{*}{ Question* } & \multicolumn{2}{c}{ At baseline } & \multicolumn{2}{c}{ Immediately after training } \\
\cline { 2 - 6 } & Average & SD & Average & SD & $p^{\#}$ \\
\hline a) Understanding how to listen to subordinates & 2.37 & 1.01 & 3.16 & 0.60 & $<0.01$ \\
b) Positive attitude toward listening to subordinates & 2.95 & 0.71 & 2.89 & 0.66 & 0.67 \\
c) Understanding how to support workers who are returning & 1.95 & 0.97 & 3.21 & 0.63 & $<0.01$ \\
$\quad$ to work after experiencing mental health problems & & & & \\
d) Understanding how to work with occupational health professionals & 2.32 & 1.00 & 3.16 & 0.69 & $<0.01$ \\
e) Understanding the psychosocial work environment & 2.47 & 0.70 & 3.11 & 0.66 & $<0.01$ \\
f) Positive attitudes toward improving the work environment & 2.63 & 0.68 & 2.84 & 0.60 & 0.22 \\
g) Understanding how to deal with stress & 1.95 & 0.91 & 3.05 & 0.71 & $<0.01$ \\
h) Being aware of and coping well with stress & 2.74 & 0.65 & 3.05 & 0.62 & 0.06 \\
\hline
\end{tabular}

*Each question was asked using a four-point response options: from 1="not at all" to 4="very well or very much". ${ }^{\#} p$ value by paired $t$-test was shown.

control group. The intervention effect was significant $(p<0.05)$. The score for the item on the friendliness of the atmosphere in the workgroup increased during the follow-up period among the intervention group while it remained stable among the control group. The intervention effect was significant $(p<0.05)$.

\section{Effects on worksite support and psychological distress}

No significant intervention effect of the web-based supervisor training on supervisor support or coworker support was observed (Table 2, $p>0.05$ ). No significant intervention effect was observed for the total score or any sub-scales of psychological distress $(p>0.05)$. The above results for intervention effects on job stressors, worksite support, and psychological distress were unchanged after adjustment for gender and age.

\section{Discussion}

Supervisors' knowledge and attitudes toward worksite mental health were improved among supervisors who received the web-based training, as we previously observed $^{14)}$. However, we observed no significant intervention effect on the scores of any scale of job stressor, worksite support, or psychological distress, despite our intention to minimize contamination of the information given by the web-based training to the nontraining group. When looking at individual items on job stressors, we observed a statistically significant intervention effect on the maintenance of worker autonomy and improvement in the friendliness of the workplace atmosphere.

Worker autonomy on task order and method of doing jobs was maintained during the follow-up period in the intervention group, while it decreased among those in the control group. Worker autonomy, together with another aspect of job control, worker participation, is considered to have a favorable effect on worker health ${ }^{17)}$. We explained the concept of the job demands-control model ${ }^{18)}$ in the web-based training, emphasizing that increased job control among workers is a key to improving psychosocial work environment and to preventing job stress. In fact, after the training, supervisors' understanding of the psychosocial work environment improved among the trained group. Supervisors who received the web-based training might have become aware of the importance of allowing subordinate workers to do their jobs with a certain degree of work autonomy. No significant intervention effect was observed for control over work pace or influence over worksite policy. Once supervisors become aware of this, it might have been easier in this particular company to increase worker autonomy, compared with other aspects of job control, since most workers engaged in sales and service jobs frequently worked independently at retail shops and with their customers outside their offices.

Workers' responses to the question on the friendliness of the atmosphere of a workgroup changed favorably among subordinate workers in the intervention group. A friendly workplace atmosphere or a supportive social climate at work is often characterized by mutual support, mutual respect, teamwork, group commitment ${ }^{19)}$, and organizational support ${ }^{20)}$. Building and maintaining a friendly workplace atmosphere is closely related to support from supervisors ${ }^{21)}$ and is considered to be important in the work of a supervisor ${ }^{6,19)}$. The web-based training also focused on keeping a better interpersonal relationship at work. This may increase supervisors' awareness and encourage their behavior in developing a friendly worksite atmosphere. Supervisors' understanding of how to listen to their subordinates also improved after the training. This finding is in agreement with those from a previous study involving a two-day 


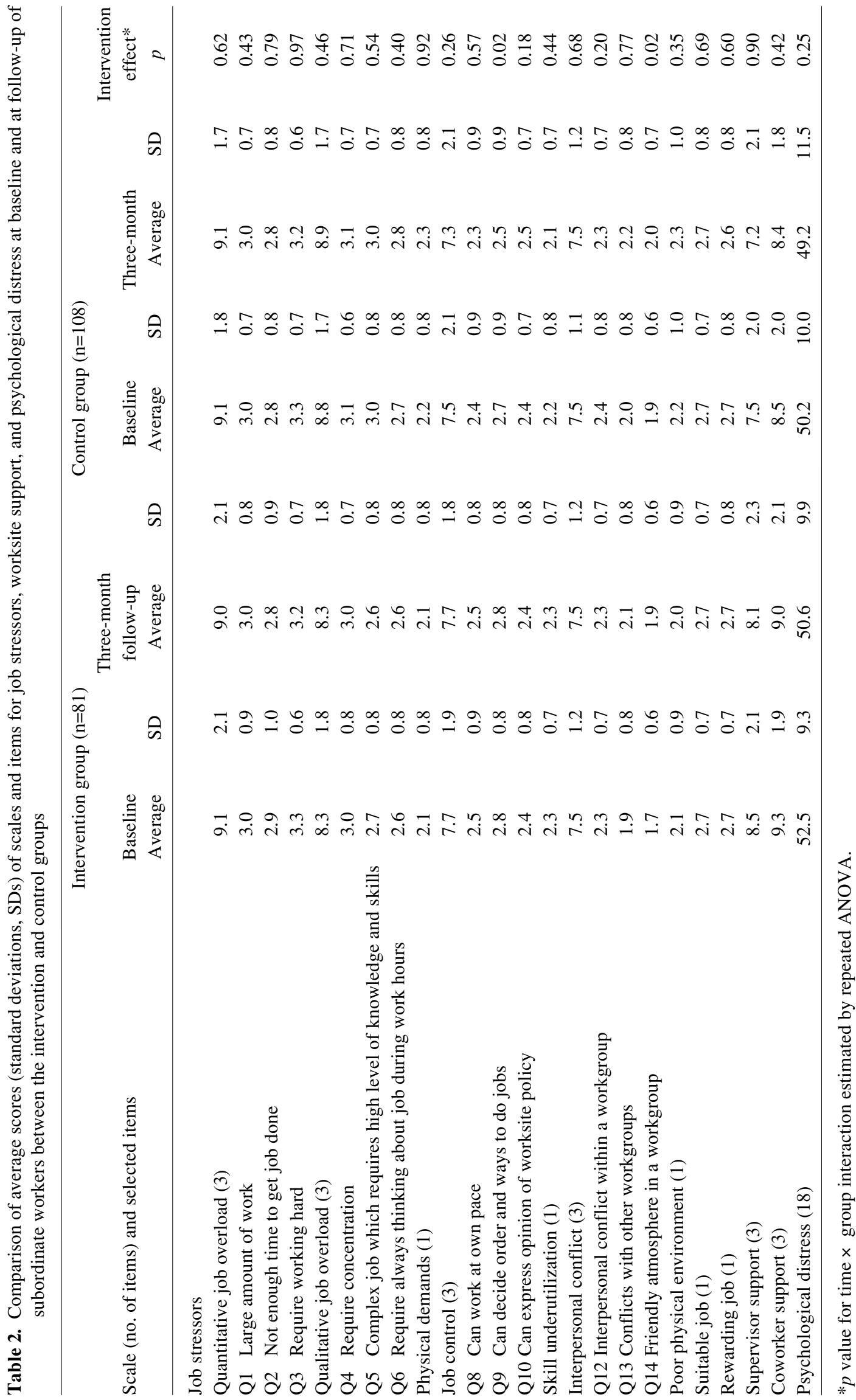


training program for supervisors that stressed listening skills $^{8,9)}$. However, in the present study we failed to show a significant intervention effect for supervisor support, while we did in our previous intervention study ${ }^{14)}$. The discrepancy may be attributable to a difference in characteristics of subjects between these two studies. First, the present sample of workers had a greater level of supervisor support at baseline than the sample of workers in the previous study. Higher levels of baseline supervisor support may result in a less clear effect of the web-based training. Second, supervisor support remained stable in the control group of the present study, while it decreased in the control group of the previous study. The effect of the web-based training may be mainly maintaining supervisor support and thus may be clearly shown only under deteriorated work conditions to decrease supervisor support. Third, the discrepancy may be attributable to the different distributions of occupations of the subjects in these two studies: sales workers in the present study and computer engineers in the previous study ${ }^{14)}$. For sales workers who work away from their workplace for most of their working hours, listening skills among supervisors, if enhanced, may still not be enough to increase perceived supervisor support.

We did not find a significant effect of the web-based training on the improvement of psychological distress among subordinate workers, despite improvement in worker autonomy and a friendly workplace atmosphere. The improvements in items of worker autonomy and friendliness of the workplace atmosphere however were small, since they were not reflected in the change in a total scale score. The information may still not be sufficient for supervisors to improve the psychosocial work environment by themselves or to reduce psychological distress among workers. The nonsignificant results may be attributable to the short followup period (three months) in the present study. Even for workers' perception of autonomy and friendliness of the workplace atmosphere, we may need to wait more before the effect of these aspects on psychological distress can be seen.

In the present study, we used workplaces as the unit for randomization to prevent contamination of information given by the web-based training to the nontraining groups. In addition, the follow-up rate of baseline workers was quite high. Thus possible bias due to attrition may be minimal. In contrast to these strengths of the present study, several limitations of the study should be considered in any interpretation of the findings. The outcome measures in this study were all subjective and self-reported. If supervisors in the intervention group had informed their subordinates that they had undergone the training, the responses of the subordinates in the intervention group to the follow-up survey could have been positively biased. While workplaces were randomly assigned to the intervention and control groups, there was a difference in characteristics of workers and some of the job stressors at baseline between the two groups, mainly because of the small number of workplaces $(n=8)$ that were randomized in this study. While the same tendency was still observed after controlling for gender and age, it is possible that some other important characteristics at baseline were different between the two groups, which might have affected the findings. We provided a relaxation training to the non-training supervisors, which may have improved their understanding of, and attitude toward, worker mental health to some extent thereby attenuating the effects of the web-based training. Finally, we failed to show a significant effect on primary or secondary outcome measures. The observed intervention effect on worker autonomy and friendliness of the workplace atmosphere may be trivial and attributable to multiple testing. Further improvements in the web-based training dealing with the psychosocial work environment and a future trial with a larger number of units for randomization are required.

Acknowledgments: The study was supported by a "Partnership between Universities and Industry Program (A)" (2001-2003) between the Japan Ministry of Education, Culture, Sports, Science and Technology and Fujitsu Infosoft Technology, Co., Ltd.

\section{References}

1) JA Winnubst, FH Marcelissen and RJ Kleber: Effects of social support in the stressor-strain relationship: a Dutch sample. Soc Sci Med 16: 475-482 (1982)

2) U Bultmann, IJ Kant, CA Schroer and SV Kasl: The relationship between psychosocial work characteristics and fatigue and psychological distress. Int Arch Occup Environ Health 75: 259-266 (2002)

3) C Dormann and D Zapf: Social support, social stressors at work, and depressive symptoms: testing for main and moderating effects with structural equations in a three-wave longitudinal study. J Appl Psychol 84: 874884 (1999)

4) C Baruch-Feldman, E Brondolo, D Ben-Dayan and J Schwartz: Sources of social support and burnout, job satisfaction, and productivity. J Occup Health Psychol 7: 84-93 (2002)

5) MA Steinhardt, CL Dolbier, NH Gottlieb and KT McCalister: The relationship between hardiness, supervisor support, group cohesion, and job stress as predictors of job satisfaction. Am J Health Promot 17: 382-389 (2003)

6) H Hiro: Stress prevention and supervisor training. Sangyo Eiseigaku Zasshi 43: 1-6 (2001) (in Japanese)

7) N Kawakami, S Araki, M Kawashima, T Masumoto and T Hayashi: Effects of work-related stress reduction on depressive symptoms among Japanese blue-collar workers. Scand J Work Environ Health 23: 54-59 (1997) 
8) S Kubota, N Mishima, A Ikemi and S Nagata: A research in the effects of active listening on corporate mental health training. J Occup Health 39: 274-279 (1997)

9) S Kubota, N Mishima and S Nagata: A study of the effects of active listening on listening attitudes of middle managers. J Occup Health 46: 60-67 (2004)

10) N Kawakami, M Kawashima, T Masumoto, K Okubo, M Hirota, N Watanabe, T Hayashi, T Haratani, N Iwata, Y Imanaka, S Araki and K Murata: "Shokuba ni okeru sutoresu taisaku-Kainyuu kenkyuu ni yoru kouka hyouka" (Preventing stress at work-the effects evaluated by intervention studies). Sanguo Igaku Journal 19: 49-53 (1997) (in Japanese)

11) A Tsutsumi, S Takao, S Mineyama, K Nishiuchi, H Komatsu and N Kawakami: Effects of a supervisory education for positive mental health in the workplace: a quasi-experimental study. J Occup Health (2005)

12) L Lenert, RF Munoz, J Stoddard, K Delucchi, A Bansod, S Skoczen and EJ Perez-Stable: Design and pilot evaluation of an Internet smoking cessation program. J Am Med Inform Assoc 10: 16-20 (2003)

13) JA Fernandez, L Vazquez, JA Daltuva, TG Robins and M Williams: Development and evaluation of an advanced training technology course within a unionbased industrial emergency response training program. Am J Ind Med 43: 429-435 (2003)

14) N Kawakami, Y Kobayashi, S Takao and A Tsutsumi: Effects of web-based supervisor training on supervisor support and psychological distress among workers: a randomized controlled trial. Prev Med 41: 471-478 (2005)

15) Japanese Ministry of Labour. Guidelines for Promoting Mental Health Care in Enterprises. Tokyo: Ministry of Labour, Japan, 2000 (in Japanese)

16) Shimomitsu T, Haratani T, Nakamura K, Kawakami N, Hayashi T, Hiro H, Arai M, Miyazaki S, Furuki K, Ohya Y, Odagiri Y. The final development of the Brief Job Stress Questionnaire mainly used for assessment of the individuals. In: Kato M, ed. The Ministry of Labour sponsored grant for the prevention of workrelated illness: The 1999 report. Tokyo: Tokyo Medical
College, 2000: 126-164 (in Japanese)

17) Karasek R and Theorell T. Healthy work. New York: Basic Books, 1990.

18) R Karasek: Job demand, job decision latitude, and mental strain: implications for job redesign. Adm Sci Q 24: 285-308 (1979)

19) CK Wilson: Team behaviors: working effectively in teams. Semin Nurse Manag 6: 188-194 (1998)

20) L Rhoades and R Eisenberger: Perceived organizational support: a review of the literature. J Appl Psychol 87: 698-714 (2002)

21) R Eisenberger, F Stinglhamber, C Vandenberghe, IL Sucharski and L Rhoades: Perceived supervisor support: contributions to perceived organizational support and employee retention. J Appl Psychol 87: 565-573 (2002)

Appendix. Table of contents of the web-based training on worksite mental health for supervisors

Introduction Scope and contents of the training

Chapter 1 The story of worker A with depression

Chapter 2 Why is mental health important in the workplace?

Chapter 3 "The Guidelines for Promoting Mental Health Care in Enterprises" by the Japanese Ministry of Health, Labour and Welfare, and roles of supervisors in occupational mental health

Chapter $4 \quad$ Listening to and advising subordinate workers and using mental health services

Chapter 5 Supporting workers who are returning to work after experiencing mental health problems

Chapter 6 Improvement of the work environment for stress prevention

Chapter 7 "Self-care" — awareness of and coping with stress

Chapter 8 Essential knowledge about mental heath Chapter 9 Summary 